\title{
Accuracy of Navigation on 3DRX Data Acquired with a Mobile Propeller C-Arm
}

\author{
Theo van Walsum ${ }^{1}$, Everine B. van de Kraats ${ }^{1}$, Bart Carelsen ${ }^{2}$, \\ Sjirk N. Boon ${ }^{3}$, Niels Noordhoek ${ }^{3}$, and Wiro J. Niessen ${ }^{1}$ \\ 1 Image Sciences Institute, University Medical Center Utrecht, Heidelberglaan 100, \\ 3508 GA Utrecht, The Netherlands \\ \{theo, everine, wiro\}@isi.uu.nl - http://www.isi.uu.nl \\ 2 Academic Medical Center Amsterdam, Medical Physics Department, \\ The Netherlands \\ 3 Philips Medical Systems, Best, The Netherlands
}

\begin{abstract}
Recently, 3DRX imaging has been combined with navigation technology, enabling direct 3D navigation, i.e. navigation on volumetric data without an explicit step to register the image data to the patient coordinate system. In this study, the accuracy of such a navigation setup is evaluated for a mobile $\mathrm{C}$-arm with propeller motion.
\end{abstract}

\section{Introduction}

Navigation on preoperative images during therapy requires registration of image data to the patient. In case of navigation on 2D fluoroscopic images, this registration is implicitly obtained by tracking both the patient and the C-arm at the moment of imaging. This approach also requires a geometric calibration and distortion correction of the C-arm images. In practice, tracking is performed by attaching a dynamic reference frame to the patient and to the C-arm. Geometric calibration is generally performed by mounting a calibration phantom, e.g. a plate with radio-opaque spheres, to the image intensifier of the $\mathrm{C}$-arm, and using the pattern of projected spheres to determine the imaging geometry and image distortion.

For 3D image guidance, the solution is less simple, as the imaging is often performed before therapy, and not in the operating room. Registration in this case is conventionally done either using markers which are rigidly attached to the patient and which are visible in the 3D images, or by performing a feature match, e.g. by matching the surface of (exposed) patient anatomy to the corresponding surface in the image. Both methods can be invasive and laborious.

A relatively new approach is to use 3D Rotational X-Ray (3DRX) data for image guidance of therapy. In 3DRX imaging, a 3D volume is reconstructed from a set of fluoroscopic images, obtained with a C-arm that rotates around the subject to image. 3DRX imaging has two distinct advantages, compared to other 3D modalities such as conventional MRI and CT: first, the images can be acquired at the operation theater, and second, the 3DRX image reconstructed 
is always at the same (or known) location with respect to a fixed position on the C-arm, which implies that, after a calibration step, the 3DRX image can be used for navigation without applying a registration step. This approach has been described earlier, and is called "direct navigation" [1,23]. As 3DRX data can also be accurately registered to other 3D imaging modalities such as MRI data [4, intra operative acquisition of 3DRX data can also be used as a replacement of the invasive and laborious conventional registration in image guided surgery on CT and MRI data.

Previously, we have reported on the accuracy of navigation of such a setup when using a fixed, ceiling-mounted C-arm [3. In this study, we investigate the navigation accuracy of a similar setup when using a mobile C-arm with propeller motion.

In Sect. 2 the concept of direct navigation is described briefly, followed by a description of our experimental setup in Sect. 3. Results and conclusions follow in Sects. 4 and 5 .

\section{Direct Navigation}

The complete process of obtaining 3DRX images for navigation consists of two calibration steps and an imaging step.

The first calibration step is required for 3DRX reconstruction from projection images. In this step, image distortion, imaging geometry and the orientation of the C-arm for each of the fluoroscopic images is determined. First, images are obtained with a bullet-grid attached to the image intensifier of the C-arm. These images are used to determine the imaging geometry (focal distance, etc.) and the image distortion, caused by the pincushion shaped detector and the earthmagnetic field. Subsequently, a special-purpose phantom is scanned to determine the positions from which each of the projection images is taken.

A second calibration step is required to determine the relation between the position of the imaged volume (image space) and some fixed position in the operation room (physical space), e.g. on the C-arm at a known location, see Fig. 1. Hereto, another special-purpose phantom, with a tracker plate attached to it and with fiducials inside, is imaged. The locations of the fiducials w.r.t. the coordinate system defined by the tracker plate are known. After imaging, the image data is imported into the navigation software and the fiducials are pinpointed in the image. Next, the positions of the pinpointed fiducials in the image are registered to the known fiducial locations in the phantom, which yields the relation between image space and the tracker plate. The relation between the $\mathrm{C}$-arm and the phantom tracker plate is determined by attaching a tracker plate to the $\mathrm{C}$-arm, and capturing the relation between the $\mathrm{C}$-arm tracker plate and the phantom tracker plate by means of a camera. This is done with the $\mathrm{C}$-arm in a known (reproducible) position, thus the relation of the image space to $\mathrm{C}$-arm space is known, and can be reused when a patient is imaged. 
Both calibration steps need to be performed before imaging of the patient. If the system is calibrated when the patient is imaged, direct navigation without an explicit registration step is possible.

When using direct navigation in practice, a dynamic reference frame must be attached to the patient, and the relation between the reference frame and the $\mathrm{C}$-arm must be stored prior to imaging. As the relation from C-arm to image space is known, because of the calibration step, the relation between the patients dynamic reference frame and the image space is known, which allows direct navigation on the 3DRX image data.

\section{$3 \quad$ Experiments}

The accuracy of direct navigation on 3DRX data has been evaluated. For imaging, a prototype mobile C-arm (Pulsera with motorized propeller facility, Philips Medical Systems, Best, The Netherlands) was used to generate the 3DRX images. Navigation was performed using a Treon StealthStation navigation system (Medtronic SNT, Louisville CO, USA) with special software that permits navigation on 3DRX images.

For the accuracy experiments, a special-purpose phantom has been used [5]. The phantom consists of 19 vertical Perspex rods with divots at the top. The locations of the divots on the rods are accurately known from the manufacturing process. The locations of the rods on the phantom have been measured with a precision of $3 \mu \mathrm{m}$. Before imaging, radiodense spheres are put on top of each of the rods, see Fig. 2 a. The center of the sphere coincides with the top of the divot, because of the way the sphere and rods are constructed. After imaging, the spheres are removed without moving the phantom, and a navigated pointer is used to touch and store the divot locations in the navigation software (Fig. 2 $b$ ), in combination with the 3DRX image of the phantom.

Afterwards, special purpose image processing software is used to determine the centers of the spheres with subpixel accuracy. Next, the set of known divot locations is registered rigidly to the sphere centers found (average RMSE for this match is $0.29 \mathrm{~mm}$ ). This set of divot locations replaces the divot locations found in the image, and is considered to be the gold standard for evaluating the positions touched with a pointer.

Using the gold standard divot locations, the target registration error (TRE), defined as the distance between gold standard divot location and the divot location as stored in the navigation system, for each of the divots can be determined. Both the mean TRE and the standard deviation of the TRE are reported.

Two experiments have been carried out in determining the accuracy: one experiment in which the $\mathrm{C}$-arm did not move in between the calibration steps and the imaging, and one experiment in which the $\mathrm{C}$-arm was moved after calibration and before imaging.

In the first experiment, after imaging of the phantom, all 19 divots were touched ten times, five times using a Passive Planar Blunt probe (Medtronic SNT, Louisville CO, USA) and five times using a Passive Planar Ball probe 


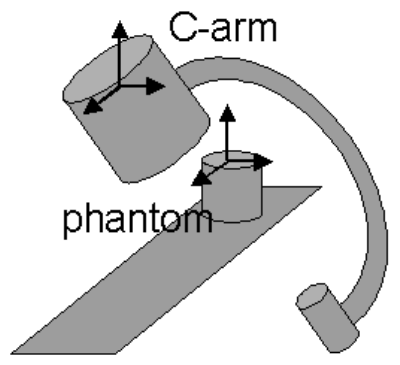

a.

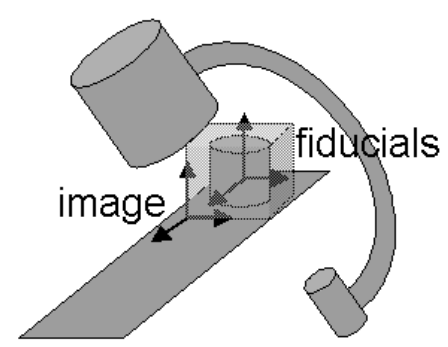

c.

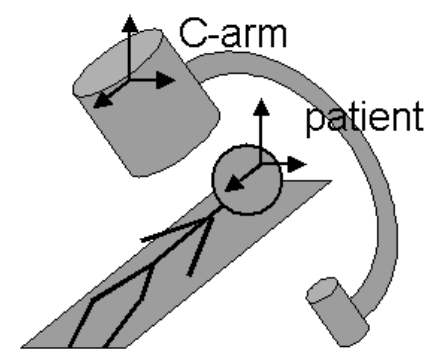

e.

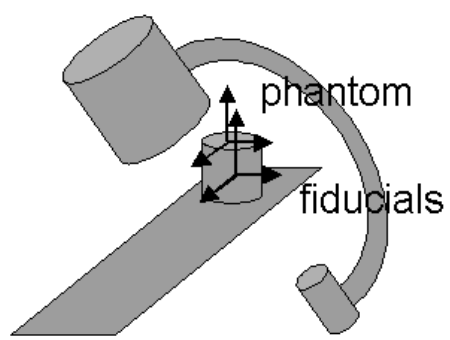

b.

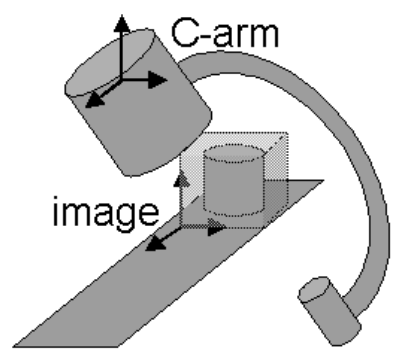

d.

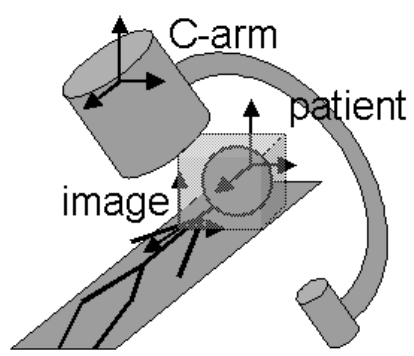

f.

Fig. 1. Transformations involved in direct navigation: a) relation between fixed position on C-arm and phantom tracker plate, captured with camera prior to imaging; b) relation between phantom tracker plate and fiducials in phantom, known by manufacturing; c) relation between fiducials in phantom, and imaged fiducials, known by registering two pointsets; d) combination of a) - c) yields relation between fixed position on C-arm, and the image coordinates; e) relation between patient reference frame and fixed position on $\mathrm{C}$-arm, captured with camera prior to imaging; f) relation between patient reference frame and image, determined from d) and e). 


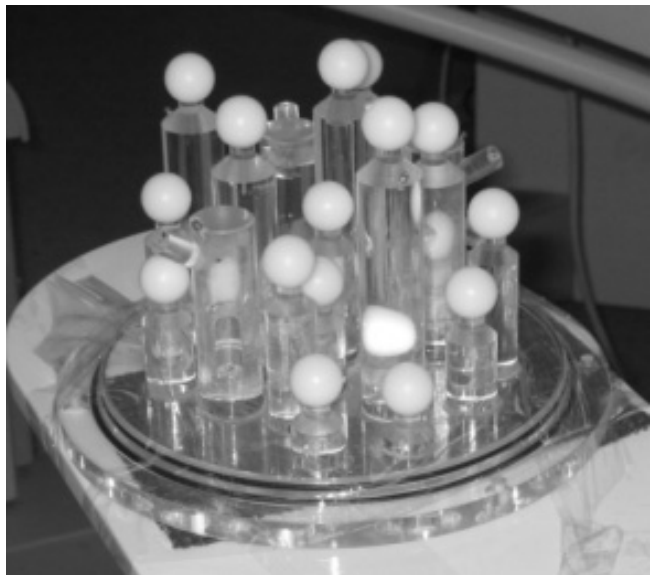

a.

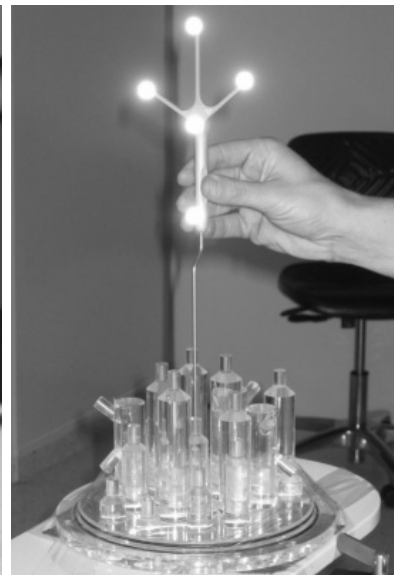

b.

Fig. 2. Phantom for accuracy experiments: a.) phantom to be imaged, with spheres on top of the rods, b.) touching of the divots after the spheres have been removed.

(Medtronic SNT, Louisville CO, USA). For each probe, three observers performed the touching of the divots, one observer three times, and the other two only once. Moving the C-arm around after calibration was expected to affect the accuracy negatively, the results of this first experiment thus should give the optimal accuracy that can be obtained with our setup.

In the second experiment, the C-arm was moved before each imaging step. The phantom was imaged six times, three times after horizontal movement, and three times after vertical movement. Calibration was done twice, once before all horizontal movement imaging, and once (because of an accident with the C-arm tracker plate) before vertical movement imaging. After each imaging step, all divots were touched twice: once by one observer, using the Passive Planar Blunt probe, and once by another observer, using the Passive Planar Ball probe.

\section{Results and Discussion}

The experiment without moving the C-arm results in a mean TRE of $0.78 \mathrm{~mm}$. This error is determined using 189 points (nineteen divots, five times with one probe, and five times with the other probe; one point was left out, because is was more than $100 \mathrm{~mm}$ off). A listing of the errors and standard deviation is shown in Table 1. There is no significant difference between the three observers in this experiment, and there is no significant difference between the two probes used.

The second experiment shows the effect of moving the C-arm around after calibration. Here, the mean TRE is $1.04 \mathrm{~mm}$ (220 points: nineteen divots, six images, two probes; eight outliers where removed from the data), which was not significantly different from the mean TRE of the C-arm that has not moved. 
Table 1. Mean TRE and standard deviation of the first experiment: no movement of the C-arm.

\begin{tabular}{lcc}
\hline Probe, Observer & $\begin{array}{c}\text { mean TRE } \\
(\mathrm{mm})\end{array}$ & $\begin{array}{c}\text { std. dev. } \\
(\mathrm{mm})\end{array}$ \\
\hline Blunt, A & 0.79 & 0.19 \\
Blunt, A & 0.68 & 0.15 \\
Blunt, A & 0.85 & 0.24 \\
Blunt, B & 0.73 & 0.16 \\
Blunt, C & 0.81 & 0.16 \\
\hline Ball, A & 0.79 & 0.25 \\
Ball, A & 0.81 & 0.20 \\
Ball, A & 0.89 & 0.33 \\
Ball, B & 0.66 & 0.23 \\
Ball, C & 0.80 & 0.25 \\
\hline Blunt, All & 0.77 & 0.19 \\
Ball, All & 0.79 & 0.26 \\
Both, All & 0.78 & 0.22 \\
\hline
\end{tabular}

Table 2. Mean TRE and standard deviation of the second experiment: horizontal and vertical movement of the C-arm; A stands for observer A with Passive Planar Blunt probe, B stands for observer B with Passive Planar Ball probe.

\begin{tabular}{lcc}
\hline Motion & $\begin{array}{c}\text { mean TRE } \\
(\mathrm{mm})\end{array}$ & $\begin{array}{c}\text { std. dev. } \\
(\mathrm{mm})\end{array}$ \\
\hline Horizontal 1, A & 0.99 & 0.18 \\
Horizontal 1, B & 0.73 & 0.24 \\
Horizontal 2, A & 1.59 & 0.18 \\
Horizontal 2, B & 1.19 & 0.13 \\
Horizontal 3, A & 1.61 & 0.50 \\
Horizontal 3, B & 1.39 & 0.39 \\
\hline Vertical 1, A & 1.26 & 0.31 \\
Vertical 1, B & 0.88 & 0.35 \\
Vertical 2, A & 0.52 & 0.23 \\
Vertical 2, B & 0.67 & 0.19 \\
Vertical 3, A & 0.82 & 0.22 \\
Vertical 3, B & 0.84 & 0.62 \\
\hline All A & 1.13 & 0.49 \\
All B & 0.95 & 0.43 \\
Horizontal, A \& B & 1.25 & 0.43 \\
Vertical, A \& B & 0.83 & 0.42 \\
All, A \& B & 1.04 & 0.47 \\
\hline
\end{tabular}

The mean TRE for navigation after horizontal motion of the C-arm is $1.25 \mathrm{~mm}$ (110 points), and the mean TRE for vertical motion is $0.83 \mathrm{~mm}$ (110 points). A listing of the errors and standard deviation is shown in Table 2 
From these results it follows that moving the C-arm around does not severely affect the navigation accuracy negatively. As approximate orientation and position of the C-arm are still the same, the 3DRX calibration probably still holds. Furthermore, the C-arm appears to be rigid enough to be moved around after calibration for navigation.

The overall accuracy of $1.0 \mathrm{~mm}$ is sufficient for many navigated surgical interventions. When these numbers are compared to the results for a ceiling-mounted C-arm (RMSE $0.7 \mathrm{~mm} \mathrm{[3]),} \mathrm{these} \mathrm{numbers} \mathrm{show} \mathrm{that} \mathrm{the} \mathrm{mobility} \mathrm{of} \mathrm{the} \mathrm{C-arm}$ system only slightly compromises the accuracy. Furthermore, similar numbers have been reported for the SIREMOBIL Iso-C ${ }^{3 \mathrm{D}}[2]$; the average errors for that $\mathrm{C}$-arm range from 1.0 to $1.6 \mathrm{~mm}$, depending on the experimental setup.

Given the propeller motion of the $\mathrm{C}$-arm, imaging and thus navigation is limited to extremities and pediatric, head and neck applications.

\section{Conclusion}

Direct navigation using a mobile C-arm with propeller motion is accurate. In our experiments, the mean TRE is $1.0 \mathrm{~mm}$, which is sufficient for many applications.

Acknowledgments. Herke-Jan Noordmans is acknowledged for his assistance in extracting the sphere centers from the 3DRX images of the phantom.

\section{References}

1. Mitschke, M., Ritter, D.: Direct navigation with an isocentric mobile C-arm. In: Computer Assisted Orthopedic Surgery. (2002) 209-211

2. Ritter, D., Mitschke, M., Graumann, R.: Markerless navigation with the intraoperative imaging modality SIREMOBIL ISO-C3D. Electromedica 70 (2002) 31-36

3. Van de Kraats, E.B., Van Walsum, T., Kendrick, L., Noordhoek, N., Niessen, W.J.: Direct navigation on 3D rotational X-ray images. In: Computer Assisted Orthopedic Surgery. (2003) 382-383

4. Van de Kraats, E.B., Van Walsum, T., Verlaan, J.J., Öner, F.C., Viergever, M.A., Niessen, W.J.: Noninvasive magnetic resonance to three-dimensional rotational Xray registration of vertebral bodies for image-guided spine surgery. Spine 29 (2004) 293-297

5. Willems, P.W.A., Noordmans, H.J., Berkelbach van der Sprenkel, J.W., Viergever, M.A., Tulleken, C.A.F.: An MKM-mounted instrument holder for frameless pointstereotactic procedures: a phantom-based accuracy evaluation. Journal of Neurosurgery 95 (2001) 1067-1074 